\title{
Erratum: Higher-order tree-level amplitudes in the nonlinear sigma model
}

\author{
Johan Bijnens, ${ }^{a}$ Karol Kampf $^{b}$ and Mattias Sjö ${ }^{a}$ \\ ${ }^{a}$ Department of Astronomy and Theoretical Physics, Lund University, \\ Sölvegatan 14A, Lund, Sweden \\ ${ }^{b}$ Institute of Particle and Nuclear Physics, Charles University, \\ V. Holešovičkách 2, Prague, Czech Republic \\ E-mail: bijnens@thep.lu.se, karol.kampf@mff.cuni.cz, \\ mattias.sjo@thep.lu.se
}

ERratum to: JHEP11(2019)074

ARXIV EPRINT: 1909.13684

In the original paper the supplementary material was incorrect and incomplete. The correct supplementary material is attached to this erratum.

Open Access. This article is distributed under the terms of the Creative Commons Attribution License (CC-BY 4.0), which permits any use, distribution and reproduction in any medium, provided the original author(s) and source are credited. 